\title{
Laparoendoscopic Approach in the Management of Abdominal Wall Hernia - Our Initial Experience from Chennai, India
}

\author{
Chandru Ravindrakumar ${ }^{1}$, Saravanan Sanniyasi ${ }^{2}$, Pervez Ahmed ${ }^{3}$, Surya Subramaniam ${ }^{4}$ \\ 1,2 Department of General Surgery, Sri Ramachandra Institute of Higher Education and Research, \\ Chennai,Tamil Nadu, India. ${ }^{3}$ Department of Colorectal Surgery, Royal Oldham Hospital, Manchester, UK, \\ ${ }^{4}$ Department of Plastic Surgery, Ganga Hospital, Coimbatore, Tamil Nadu, India.
}

\section{ABSTRACT}

\section{BACKGROUND}

Incisional hernias are a common occurrence following midline laparotomy. Numerous techniques have been employed in the management of this common condition. Randomized trials and evidence based medicine help us in deciding what the optimal treatment is in a particular condition. This is often not possible in case of hernia due to the heterogeneity of population. Laparoscopy is gaining popularity in the management of this condition as results are comparable to open surgery with lesser post-operative pain. The placement of mesh intraperitoneally as in laparoscopic intraperitoneal onlay mesh repair has its own disadvantage such as mesh erosion into viscera. Placement of mesh endoscopically as an onlay repair seems to be a good option, more so in the developing world, considering the prohibitive cost of composite mesh. The purpose of this study was to analyze the technical ease, reproducibility, outcome, postoperative pain and complications of laparoendoscopic hybrid technique in the management of abdominal wall hernia.

\section{METHODS}

This technique combines the benefits of minimal invasive surgery and ease of onlay mesh repair, while reducing wound related problems associated with open surgery. It involves first a laparoscopic approach in entry, reducing the contents, visualizing the sac followed by endoscopic dissection using the same trocar anterior to abdominal wall in the subcutaneous plane. This is followed by sac dissection, suturing the abdominal wall and placing an onlay mesh

\section{RESULTS}

Fifteen patients have been operated; no subcutaneous drains were placed. A polypropylene onlay mesh was used and fixation done with suturing. No major complications were seen. There was no subcutaneous emphysema.

\section{CONCLUSIONS}

The laparoendoscopic hybrid technique offers advantage of being minimally invasive and having lower wound related complications, with early ambulation and decreased hospital stay. It is also technically easier to suture the defect and allow complete excision of the sac. However a randomized trial is necessary and larger series are required to compare the results with other established techniques.

\section{KEY WORDS}

Incisional Hernia, Laparo - Endoscopic - Onlay Mesh, Surgical Techniques, SCOLA
Corresponding Author: Dr. Saravanan Sanniyasi No. 1, Ramachandra Nagar, Porur, Chennai - 600116, Tamil Nadu, India.

E-mail:

saravanan.s@sriramachandra.edu.in

DOI: $10.14260 /$ jemds/2021/496

How to Cite This Article: Ravindrakumar C, Sanniyasi S, Ahmed P, et al. Laparoendoscopic approach in the management of abdominal wall hernia our initial experience from Chennai, India. J Evolution Med Dent Sci 2021;10(31):24222426, DOI: 10.14260/jemds/2021/496

Submission 21-12-2020,

Peer Review 24-05-2021,

Acceptance 31-05-2021,

Published 02-08-2021.

Copyright (C) 2021 Chandru Ravindrakumar et al. This is an open access article distributed under Creative Commons Attribution License [Attribution 4.0 International (CC BY 4.0)] 


\section{BACKGROUND}

Ventral and incisional hernias have become a major part of everyday clinical practice of a surgeon. Incisional hernia is reported in 10 to $30 \%{ }^{1}$ of laparotomy incisions due to various reasons and contributes to substantial morbidity with increased hospital expenses. Within first two years of primary surgery, $50 \%$ of incisional hernia occurs which increase to 74 $\%$ after 3 years. The recurrence of incisional hernias after 5 years in only open anatomical repair is $17.1 \%$ which reduced to $12.3 \%$ with mesh repair and still lower in laparascopic mesh repair to $10.6 \% .^{2}$

Laparoscopic ventral and incisional hernia repair has gained popularity over recent years. It has been accepted as a procedure with good safety margins and reproducibility. It involves placement of a mesh intraperitoneally. The laparoscopic approach offers a perioperative complication rates comparable to open surgery with a decrease in wound infection rates, haemorrhagic complications and mesh infections. There is also better delineation of Swiss cheese defects. The main disadvantage of laparoscopy is contact of the mesh with bowel and the difficulty of retrieval of mesh following infection and fistulation. There is also a prohibitive cost of the intraperitoneal mesh when compared to the polypropylene mesh. Though many improvements to the mesh are being made, reports of bowel adhesions, mesh migration ${ }^{3}$ and fistulation ${ }^{4}$ have been reported.

In an open incisional hernia repair the mesh is placed onlay, inlay or sublay with no definite consensus on the ideal position of the mesh. A meta-analysis comparing onlay versus sublay incisional hernia repair found a marginally favourable decrease in the recurrence rate with a sublay repair if one study was omitted. 5 However, there was a high level of heterogeneity and lot of variables like mesh overlap and surgeon experience were not taken into account. It is often outweighed by the more cumbersome dissection, time, bleeding and difficulty of the procedure.

Laparoendoscopic onlay mesh repair combines the benefits of minimally invasive approach and an onlay mesh repair.

\section{Objectives}

The purpose of this study was to analyze the technical ease, reproducibility, outcome, postoperative pain and complications of laparoendoscopic hybrid technique in the management of abdominal wall hernia.

\section{METHODS}

After obtaining the Institutional Ethics Committee's clearance (IEC ref no. IEC-NI/16/APR/52/24, a prospective analysis of patients undergoing laparoendoscopic approach for the management of abdominal wall hernia at a tertiary institute was conducted. All patients above the age of 18 yrs. who presented to Sri Ramachandra Medical College \& Research Institute, with a ventral or midline incisional hernias having a domain M2 (epigastric region), M3 (umbilical) and M4 (infraumbilical) with maximum defect size of $8 \mathrm{~cm}$ were included in the study.

Patients presenting with complications of incisional hernia, such as obstruction and strangulation were excluded from the study. Hernias where the skin was ulcerated and extremely thinned out or very lax requiring an abdominoplasty were excluded. Patients with chronic obstructive pulmonary disease and poor pulmonary function or when the patient is not an ideal candidate for general anesthesia were also excluded from the study. A defect size of more than $8 \mathrm{~cm}$ which necessitated to perform a component separation technique was also excluded from the study. The study was done between August 2016 and August 2017.

All patients were investigated with routine blood tests and ultrasound abdomen was done to rule out concomitant intraabdominal pathology and also measure the defect size. A total of 15 patients were included in the study which included 8 incisional hernias, ${ }^{6}$ paraumbilical hernias and one was fatty herniation of linea alba.

All underwent laparoendoscopic hernia repair with mesh reinforcement under general anaesthesia. Patient was placed in supine position. A small sandbag was placed under the buttock opposite the monitor and a tilt was given. The trocar placement is as for a conventional laparoscopic intraperitoneal mesh placement depending on the site of the hernia. This can be placed either in the anterior axillary line or in the upper abdomen or lower abdomen depending on the site of the hernia. The trocar is placed as far as possible from the defect. The initial entry was made following the installation of pneumoperitoneum using a veress needle at the Palmer's point as the hernia was predominantly in the midline. This was followed by introduction of a $10 \mathrm{~mm}$ port for camera and two $5 \mathrm{~mm}$ working ports. The next step was reduction of contents and adhesiolysis as deemed necessary. When the content was bowel sharp dissection and judicious use of electrocautery was followed. After complete assessment of the defect size and the number of defects, same trocars were withdrawn and placed in the plane between the anterior rectus sheath and subcutaneous tissue, as in conventional open surgery. Carbon dioxide insufflation was carried out at 10 - 12 $\mathrm{mm} \mathrm{Hg}$ similar to laparoscopic procedure. ETCO2 was monitored continuously. The endoscopic dissection was performed with the camera until a pocket was created for subsequent trocar insertion. With the use of harmonic scalpel flap was raised on all sides just sufficient to have a $5 \mathrm{~cm}$ overlap across all edges of the defect. This was done with care as the planes open up with very minimal dissection. The sac was clearly visible and completely dissected all around. We preferred to excise the sac completely. This step is not feasible in laparoscopic ventral hernia repair and may account for seroma formation. The multiloculated sac may also contain devitalized omentum which might act as a nidus for mesh infection.

Once the sac was dissected completely, the defect was closed with Polydioxanone 1-0 or barbed suture. Suturing the defect onlay is much simpler than closing the defect from inside. It is very similar to working on a simulator. A mesh was framed with a $5 \mathrm{~cm}$ expansion on all directions from the edge of the defect. The polypropylene mesh was tailored calculating the original defect size with a $5 \mathrm{~cm}$ overlap all around. 


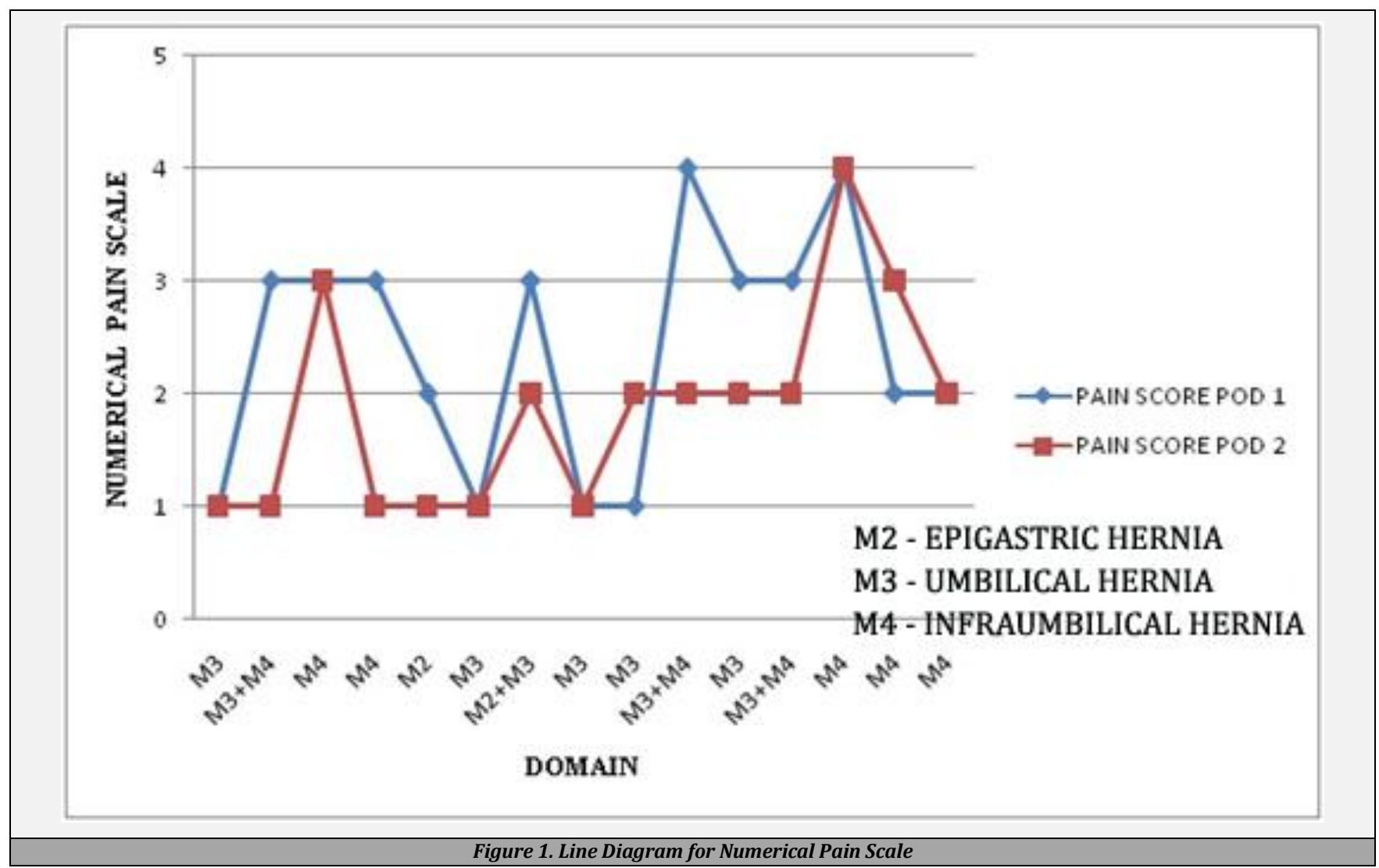

This mesh was introduced and anchored to the anterior abdominal wall with 2 - 0 PDS. No drains were placed. The post-operative pain, length of stay, wound complications were recorded and documented.

\section{Statistical Analysis}

All the data were filled in a Microsoft Excel sheet and analyzed. Descriptive statistics of quantitative data was presented as mean and standard deviation.

\section{RESULTS}

Our study had 15 cases of hernia, who underwent laparoendoscopic hernia repair with mesh reinforcement. Of this, 8 were incisional hernias, 6 were paraumbilical hernias and one was fatty herniation of linea alba. There were 9 males and 5 females in our study with median age of 48 years (range 32 to 64). The defect size ranged from $2 \mathrm{~cm}$ to $8 \mathrm{~cm}$ with a mean of $5.23 \mathrm{~cm}$. The mean operating time was 112 minutes. There was no intraoperative complication and no conversion to open surgery.

The postoperative pain was analyzed using the numerical pain scale which was significantly low (mean score on POD $2=$ 1.87 and standard deviation of 0.92) [Fig.1] with better patient satisfaction and early discharge from hospital (less than 3 days). No significant seroma which required aspiration was noted. The most significant benefit of this technique is the very low incidence of wound infection and wound related complications as one might expect in open repairs. One patient who was morbidly obese developed skin discoloration at the summit which settled after application of binder. Early ambulation with abdominal compression binders was advised for 4 to 6 weeks. There was no mesh related complications. In the follow up of 2 years, there was no recurrence.

\section{DISCUSSION}

Laparoscopic repair of incisional and ventral hernia has become common and placement of intraperitoneal onlay mesh has gained popularity due to its reproducibility. The main advantages of laparoscopic repair are the shorter hospital stay, better delineation of the defect and lesser wound related infection. However, there have been increasing reports of mesh related complications, bowel adhesions, fistulations and erosion into bladder.

A meta-analysis of randomized control trial comparing open and laparoscopic ventral and incisional hernia repair ${ }^{6}$ found no difference in perioperative complications such as seroma formation, but there was a decrease in wound infection and mesh infection in the laparoscopic group. There was no difference in hernia recurrence between laparoscopic and open repair with mesh. Unlike in other laparoscopic surgeries post-operative pain is significantly higher in the laparoscopic incisional hernia, which is attributed to transfascial sutures. ${ }^{7}$

Onlay and sublay repairs of incisional hernias are well known techniques in open incisional hernia. Onlay technique, originally described by Chevrel, 8 involves placement of a mesh on the anterior rectus fascia after dissection of the subcutaneous tissue and approximation of the edges. It is easier to perform, less time consuming and reproducible. Sublay position of mesh is more technically challenging and can be difficult in extensive adhesions and infection. The 
sublay placement might not be feasible in all incisional hernias especially in the lower segment, as the posterior fascia is defective.

A meta-analysis of sublay versus onlay showed no difference in recurrence. ${ }^{5}$ Sublay repair had lesser wound infection than onlay repair. Differences in seroma formation, postoperative pain could not be assessed due to heterogeneity in placement of drain. Also, there was no performance of ultrasound in any of the trials. Hence it was difficult to conclude which was better in terms of outcome and both techniques remained acceptable. The superficial placement of the mesh and ease of dissection makes onlay repair a favoured technique by many. It is also less time consuming. However extensive dissection in this plane compromises the blood supply and leads on to more wound related complications. There is also an increasing incidence of extensive seroma formation. The retrorectus mesh placement has better outcome in terms of mesh related complication possibly due to increased vascularity. However at times it can be technically challenging, time consuming and may involve extensive dissection. Onlay repair continues to be a favored technique due to its reproducibility and shorter learning curve.

To overcome the drawback of wound related complications and extensive dissection in the placement of an onlay mesh G. Champault ${ }^{9}$ presented 15 cases of Video parietoscopic surgery. However the defect was closed with sutures and no mesh was placed. He used $\mathrm{CO}_{2}$ for progressive insufflation. Several techniques involving endoscopic video and conventional instruments have long been used by plastic surgeons.

An exclusive endoscopic approach involving $\mathrm{CO}_{2}$ insufflation and placement of mesh in the preperitoneal space was described by Juan Bellido et al. ${ }^{10}$ wherein he had placed a composite mesh to bridge the defect. This technique does not offer any benefit in terms of cost when compared to intraperitoneal onlay mesh and the minimal dissection done does not guarantee significant overlap of mesh over the defect of a minimum of $5 \mathrm{~cm}$ as recommended.

Composite meshes are used in laparoscopic incisional hernia. These help by preventing adhesions on the surface, which is in contact with the intestine. But the cost of the mesh is significantly higher than the polypropylene mesh used for open repair. With the intraperitoneal placement of the mesh, there are always instances of fistulation ${ }^{4}$ and mesh migration. ${ }^{3}$

C D Narayanan et al. ${ }^{11}$ described a hybrid technique of laparoendoscopy. This combined the benefit of laparoscopy interms of reduction of the contents and adhesiolysis, which allow us to perform even in irreducible hernias followed subsequently by endoscopic dissection, excision of the sac in toto, closure of the defect with non-absorbable sutures and tailoring the mesh to have a $5 \mathrm{~cm}$ overlap in all directions. This technique has been subsequently popularized by $\mathrm{C}$. Claus et al. ${ }^{12}$ as subcutaneous onlay laparoscopic approach (SCOLA). This however is confined to the pre aponeurotic space. The onlay placement by this technique reduces the incidence of wound related complications. However this technique cannot be used when there is laxity of skin or grossly thinned out skin or in large defects which may necessitate a component separation technique. This has also the propensity to compromise the perforator vessels which may lead on to devitalized skin. The subcutaneous dissection might also predispose to seroma formation. We had one case of discoloration of the overlying skin but did not require debridement or excision. Seroma formation was quantifiable in two cases but did not require aspiration and settled with application of abdominal binder.

Other alternatives to pre aponeurotic techniques have also been described. The most popular technique is MILOS (mini / less open sublay) described by W. Reinpold ${ }^{13}$ wherein the surgeon places a large mesh into the retro muscular plane through a small periumbilical skin incision. This technique is highly demanding and has a steep learning curve. Damage to the umbilical perforators might lead to necrosis of the umbilical skin. Modification of this technique into EMILOS (Endoscopic MILOS) by Schwarz et al. ${ }^{14}$ where hybrid method of combining the small periumbilical incision with an endoscopic vision for the dissection of retro muscular plane and placement of large mesh under vision was achieved. However this technique not only requires a long learning curve but also when the peritoneum gets opened, there will be loss of extra peritoneal space which complicates the learning curve.

Rives stoppa (RS), an age old technique has been revived using endoscopic means by Belyanski et al. ${ }^{15}$ for retro muscular mesh placement. This is particularly useful for midline ventral hernias with a defect of size not exceeding 6 $\mathrm{cm}$ and not requiring component separation. However this requires extensive dissection, disruption of intact posterior rectus sheath and is technically challenging. Defect in the peritoneum during midline cross over makes the procedure more cumbersome. The extension of extended totally extra peritoneal repair (E-TEPP RS) is an addition of TAR which allows for closure of larger size defects. This allows for midline closure with ease, a retro muscular placement of the mesh and providing more functionality of the abdominal wall. Poor selection of cases and a steep learning curve is acting as a detriment to this technique gaining popularity. Social media and rapid advancement in the field of abdominal wall reconstruction is making evidence based medicine a remote possibility.

In laparoscopic intra peritoneal onlay mesh (IPOM) repair technique, the mesh is placed over the defect and secured to the peritoneum from inside. This technique poses a higher rate of recurrence when the abdominal wall defect is more than 5 $\mathrm{cm}$. Also the location of the hernia, proximity to bony structures and tension in closing the gap plays a vital role in recurrence. While dealing with larger hernias with huge sac, the major disadvantage of leaving behind the sac, possibly contributes to seroma formation and the retained omental bits in multiloculated sac might act as a nidus for infection. The intraperitoneal placement of mesh is also a potential surface for adhesions, bowel obstruction and fistulation, which is not seen in onlay mesh repair

The laparoendoscopy approach aims to combine the benefits of minimally invasive surgery, which are better delineation of the defect, complete dissection of sac, lesser hospital stay, decrease in wound related infection and mesh infections, with the benefits of open onlay mesh repair, which are ease of repair, lower cost of the mesh and absence of contact with intestines. As there are no transfascial sutures, the postoperative pain is also significantly low.

The cost of the onlay mesh is also significantly lower than a composite mesh. Wound related infections and mesh infections are found to be low as it is minimal access technique. This technique might not be feasible in patients who have loose redundant skin requiring an abdominoplasty.

Issue 31 / Aug. 02, 2021 


\section{CONCLUSIONS}

Laparoendoscopy / SCOLA offers technical ease, low cost, easy reproducibility and good visibility of contents making it a suitable alternative. However, a larger series with randomization and standardization of the size of the defect is required.

Data sharing statement provided by the authors is available with the full text of this article at jemds.com.

Financial or other competing interests: None.

Disclosure forms provided by the authors are available with the full text of this article at jemds.com.

\section{REFERENCES}

[1] Heger P, Feißt M, Krisam J, et al. Hernia reduction following laparotomy using small stitch abdominal wall closure with and without mesh augmentation (the HULC trial): study protocol for a randomized controlled trial. Trials 2019;20(1):738.

[2] Kokotovic D, Bisgaard T, Helgstrand F. Long-term recurrence and complications associated with elective incisional hernia repair. JAMA 2016;316(15):1575-82.

[3] Ripetti V, La Vaccara V, Angelini E, et al. Mesh infection and migration after umbilical hernia repair. Surgical Science 2013;4:421-5.

[4] Sahoo MR, Bisoi S. Polypropelene mesh eroding transverse colon following laparoscopic ventral hernia repair. J Minim Access Surg 2013;9(1):40-1.

[5] Timmermans L, De Goede B, Van Dijk DM, et al. Metaanalysis of sublay versus onlay mesh repair in incisional hernia surgery. Am J Surg 2014;207(6):980-8.

[6] Forbes SS, Eskicioglu C, McLeod RS, et al. Meta-analysis of randomized controlled trials comparing open and laparoscopic ventral and incisional hernia repair with mesh. Br J Surg 2009;96(8):851-8.

[7] Beldi G, Wagner M, Bruegger LE, et al. Mesh shrinkage and pain in laparoscopic ventral hernia repair: a randomized clinical trial comparing suture versus tack mesh fixation. Surg Endosc 2011;25(3):749-55.

[8] Chevrel JP. Traitement des grande éventrations médianes par plastie en paletot et prothèse. La Nouvelle Presse Medicale 1979;8(9):695-6.

[9] Champault G, Catheline JM, Barrat C. Video-parietoscopic surgery of the abdominal wall. A study of 15 cases. Chirurgie 1998;123(5):474-7.

[10] Luque JB, Luque AB, Valdivia J, et al. Totally endoscopic surgery on diastasis recti associated with midline hernias. The advantages of a minimally invasive approach. Prospective cohort study. Hernia 2015;19(3):493-501.

[11] Narayanan CD, Sanniyasi S, Ahmed P. 24th International Congress of the European Association for Endoscopic Surgery (EAES) Amsterdam, The Netherlands, 15-18 June 2016. Surg Endosc 2017;31:462-523.

[12] Claus CMP, Malcher F, Cavazzola LT, et al. Subcutaneous Onlay Laparoscopic Approach (SCOLA) for ventral hernia and rectus abdominis diastasis repair: technical description and initial results. Arq Bras Cir Dig 2018;31(4):e1399.

[13] Reinpold W. Endoskopisch totalextraperitonealer transhernialer sublay-bauchwand hernienverschluss in single-port-technik. In: Schumpelick V, Arlt G, Conze J, et al, eds. Hernien, $5^{\text {th }}$ edn. Stuttgart: Thieme 2015:301-4.

[14] Schwarz J, Reinpold W, Bittner R. Endoscopic mini/less open sublay technique (EMILOS)-a new technique for ventral hernia repair. Langenbecks Arch Surg 2017;402(1):173-80.

[15] Belyansky I, Daes J, Radu VG, et al. A novel approach using the enhanced-view totally extraperitoneal (eTEP) technique for laparoscopic retromuscular hernia repair. Surg Endosc 2018;32(3):1525-32. 